\title{
ARTICLE OPEN Exploring patients' views of primary care consultations with contrasting interventions for acute cough: a six-country European qualitative study
}

\author{
Sarah Tonkin-Crine ${ }^{1,10}$, Sibyl Anthierens ${ }^{2,10}$, Nick A Francis ${ }^{3}$, Curt Brugman $^{4}$, Patricia Fernandez-Vandellos ${ }^{5}$, Jaroslaw Krawczyk $^{6}$, \\ Carl Llor ${ }^{7}$, Lucy Yardley ${ }^{8}$, Samuel Coenen ${ }^{2}$, Maciek Godycki-Cwirko ${ }^{6}$, Christopher C Butler ${ }^{3}$, Theo JM Verheij ${ }^{4}$, Herman Goossens ${ }^{2}$, \\ Paul Little ${ }^{1}$ and Jochen W Cals ${ }^{9}$ on behalf of the GRACE INTRO team
}

BACKGROUND: In a pan-European randomised controlled trial (GRACE INTRO) of two interventions, (i) a point-of-care C-reactive protein test and/or (ii) training in communication skills and use of an interactive patient booklet, both interventions resulted in large reductions in antibiotic prescribing for acute cough.

AIMS: This process evaluation explored patients' views of primary care consultations using the two interventions in six European countries.

METHODS: Sixty-two interviews were conducted with patients who had participated in the GRACE INTRO trial. Interviews were transcribed verbatim and translated into English where necessary. Analysis used techniques from thematic and framework analysis. RESULTS: Most patients were satisfied with their consultation despite many not receiving an antibiotic. Patients appeared to accept the use of both intervention approaches. A minority, but particularly in the trial arm with both interventions, reported that they would wait longer before consulting for cough in future.

CONCLUSIONS: Patients perceived that both interventions supported the general practitioner's (GP's) prescribing decisions by helping them understand when an antibiotic was, and was not, needed. Patients consulting with acute cough had largely positive views about the GP's enhanced communication skills, which included understanding their concerns, and the use of a near-patient test as an additional investigation.

npj Primary Care Respiratory Medicine (2014) 24, 14026; doi:10.1038/npjpcrm.2014.26; published online 17 July 2014

\section{INTRODUCTION}

The management of respiratory tract infections (RTIs) in primary care is often discussed in relation to unnecessary antibiotic prescribing. $^{1}$ In light of the association between antibiotic prescribing and resistance, many interventions have been trialled in order to promote more prudent antibiotic prescribing.

Interventions have been targeted at both general practitioners (GPs) and patients, with mixed results. ${ }^{2,3}$ Reviews have found that multi-component rather than single-component interventions lead to greater reductions in antibiotic prescribing and that interventions that contain an educational component are more effective. ${ }^{2,4,5}$ More recent studies have found that point-of-care tests and communication skills are components that can also meaningfully influence prescribing. ${ }^{6-9}$ Point-of-care testing for RTIs can help a GP to distinguish between infections that are likely to be self-limiting and those that may benefit from antibiotic treatment. ${ }^{6,7}$ Interventions that include communication skills training assist GPs in explaining a non-prescribing decision to patients in a way that is understandable and acceptable. ${ }^{8,9}$

While such interventions have been found to be effective in quantitative studies, qualitative methods are best suited to exploring the feasibility and acceptability of interventions from the perspectives of the intended users. Qualitative studies alongside trials are useful in exploring why an intervention was effective or not. ${ }^{10,11}$ Previous research has examined GPs' views of interventions and their preferences between different types of intervention. ${ }^{12-14}$ Qualitative work with patients has most often focused on patients' views of antibiotics and resistance in general. ${ }^{15,16}$ However, some studies have found that patients are usually happy to have point-of-care tests carried out and that parents of young children value booklets as a way of obtaining information when consulting for RTIs. ${ }^{17,18}$

This study forms part of a process evaluation of a randomised controlled trial (RCT). The GRACE INTRO (INternet TRaining for antibiOtic use) trial was a large, multi-country factorial cluster RCT examining the effectiveness of two interventions aimed at decreasing GP antibiotic prescribing for acute cough. ${ }^{19}$ Use of online communication skills training together with an interactive patient booklet and a point-of-care C-reactive protein (CRP) test was evaluated in a $2 \times 2$ factorial design across six European countries. GPs were trained in the use of both interventions via a web-based program that was developed by piloting the

\footnotetext{
${ }^{1}$ Academic Unit of Primary Care and Population Sciences, University of Southampton, Southampton, UK; ${ }^{2}$ Vaccine \& Infectious Disease Institute (VAXINFECTIO), University of Antwerp, Antwerp, Belgium; ${ }^{3}$ Cochrane Institute of Primary Care and Public Health, Cardiff University, Cardiff, UK; ${ }^{4}$ Julius Centre for Health Sciences and Primary Care, University Medical Center Utrecht, Utrecht, The Netherlands; ${ }^{5}$ Applied Research in Respiratory Diseases, Hospital Clinic of Barcelona, Barcelona, Spain; ${ }^{6}$ Department of Family and Community Medicine, Medical University of Lodz, Lodz, Poland; ${ }^{7}$ Primary Care Centre Jaume I, University Rovira i Virgili, Tarragona, Spain; ${ }^{8}$ Academic Unit of Psychology, University of Southampton, Southampton, UK and ${ }^{9}$ Department of General Practice, Maastricht University, Maastricht, The Netherlands.

Correspondence: S Tonkin-Crine (sktc1007@soton.ac.uk)

${ }^{10}$ These authors contributed equally to this work.

Received 17 February 2014; revised 7 May 2014; accepted 26 May 2014
} 
Table 1. The numbers of patients recruited in each country and within each intervention arm

\begin{tabular}{|c|c|c|c|c|c|c|c|}
\hline GP training in use of CRP test & 4 & 2 & 4 & 4 & 4 & 2 & 20 \\
\hline Total & 11 & 8 & 12 & 12 & 12 & 7 & 62 \\
\hline
\end{tabular}

interventions in all countries. ${ }^{20}$ Both interventions achieved important reductions in antibiotic prescribing for respiratorytract infections; GPs with CRP training prescribed fewer antibiotics than those without (33\% vs. $48 \%$ ) and GPs with communication training prescribed fewer antibiotics than those without $(36 \%$ vs. $45 \%)^{19}$

The aim of this study was to explore patients' experiences of consulting a GP participating in the GRACE INTRO trial for an acute cough when at least one of two interventions was used. In particular we aimed to explore any differences in patients' views between the intervention arms of the trial. A qualitative study with GPs was carried out in tandem and is reported elsewhere (S Anthierens, S Tonkin-Crine, J Cals, S Coenen, L Yardley, L Brookes-Howell et al., personal communication).

\section{MATERIALS AND METHODS}

This was a qualitative study with semi-structured interviews carried out with patients who participated in the intervention arms of the GRACE INTRO RCT. The GRACE INTRO RCT was trialled in six countries: Belgium, England, The Netherlands, Poland, Spain and Wales. These countries provided large variation in population (Northern, Eastern and Southern European), primary care access (same day versus slower), mechanism of payment (private, public, fee-for-service, capitation, incentives) and contextual factors (for example, in some countries a 'sick note' from a doctor is required for any sickness absence). GPs recruited 4264 adult patients from 246 GP practices who had presented to primary care with acute cough. General practices were randomised to one of four arms: (i) control, (ii) communication skills and patient booklet, (iii) CRP test or (iv) both interventions.

Patients were recruited from those who had taken part in the GRACE INTRO trial and who had given written consent to being interviewed when they had entered the RCT. Most patients gave consent to be interviewed and participants were selected based on their characteristics and invited to take part in the qualitative study. Only patients from three of the four intervention arms were invited to take part, as patients in the control arm had received no intervention and therefore could not comment on either of the interventions of interest. We aimed to recruit 15-20 patients per arm in order to compare patients' views between groups. We aimed to obtain maximum variation within the samples to capture the views of patients of both sexes, various ages and from each of the six countries. Patients were contacted by phone or email, most often within 2 weeks of their initial consultation. Researchers aimed to sample up to 12 patients from each country in order to produce an informative but manageable data set. All patients contacted completed an interview. It was not possible to continue interviewing until data saturation was achieved because the delay between interview, translation and transcription made this impractical. Researchers were satisfied that similarities across intervention arms and countries indicated data saturation for the key themes reported here.

Six experienced primary care researchers (ST-C, SA, CB, PF-V, JK, CL) carried out telephone interviews following a semi-structured interview guide between February and May 2011 (Supplementary Appendix). Questions asked about what had happened during the consultation and patients' perceptions about the outcome. Patients were asked about intervention components depending on which arm of the trial their practice had been randomised to. Patients were unaware of the trial results at the time of interview. Interviews were audio recorded and transcribed verbatim. Interviews in England and Wales were carried out in English. In the remaining four countries interviews were carried out in Dutch
Table 2. Patient characteristics across the three intervention arms

Patient gender Patient age

N (\%) female Mean (years) Range (years)

\begin{tabular}{lccc}
\hline CRP training & $10(50 \%)$ & 51.5 & $25-78$ \\
$\begin{array}{l}\text { Communication training } \\
\text { and booklet }\end{array}$ & $13(59 \%)$ & 54.5 & $19-79$ \\
$\begin{array}{l}\text { Both interventions } \\
\text { All interviews }\end{array}$ & $10(50 \%)$ & 54.9 & $20-76$ \\
\hline
\end{tabular}

Abbreviation: CRP, C-reactive protein.

(Belgium, Netherlands), Spanish (Spain) and Polish (Poland). Transcripts were translated into English in the country of origin. English transcripts were checked by the original interviewer, who was fluent in both English and the language in which the interview was carried out. Interviewers ensured that each transcript represented the original interview.

Interview data were analysed using thematic and framework analysis. ${ }^{21,22}$ NVivo 9 software was used to facilitate data analysis. Thematic analysis allowed researchers to take an initial inductive approach, which reduced the risk of interpretations being influenced by the researchers' initial ideas or previous literature. Techniques from framework analysis allowed the large data set to be charted in order to make comparisons between different intervention arms and countries. ST-C and SA did independent, line-by-line coding of a selection of 26 interviews each. Two sets of initial themes were created, which were compared, discussed by ST-C, SA, NAF and JWC and revised to create a final thematic framework. Four themes and 12 subthemes were initially identified. ST-C used the framework to analyse the remaining 36 interviews. Any data that did not fit under the existing themes was coded as a new item and this resulted in two additional subthemes. The final thematic framework was amended and reduced to three themes with nine subthemes. All themes and subthemes were renamed at least once during the analysis process.

\section{RESULTS}

Sixty-two patients were interviewed, with similar numbers recruited across intervention arms and countries (Table 1). Interviews lasted from 4 to $18 \mathrm{~min}$, with a median of $10 \mathrm{~min}$. Patient characteristics were similar between intervention arms (Table 2).

Eighteen patients (29\%) had received an antibiotic as a result of their initial consultation, $6(9 \%)$ had received a delayed prescription and $38(62 \%)$ had not received an antibiotic. Patients in the communication arm had received fewer prescriptions (Table 3 ). Five patients (12.5\%) had not received a CRP test even though they were in a CRP intervention arm. In three cases this was due to the doctor using their own discretion about whether or not it was needed. The remaining two cases, in the Netherlands, had had blood sent to a laboratory.

Three themes were identified that were relevant to all patients. Any differences identified between the views of patients in different intervention arms or from different countries are mentioned under each theme. 
Table 3. The number of patients receiving an antibiotic prescription or delayed prescription in each intervention arm

\begin{tabular}{|c|c|c|c|}
\hline & Antibiotic prescription & Delayed prescription & Any prescription \\
\hline GP training in use of CRP test $(n=20)$ & $7(35 \%)$ & $1(5 \%)$ & $8(40 \%)$ \\
\hline Total & 18 & 6 & 24 \\
\hline
\end{tabular}

1. Theme 1: Patients' reasons for consulting

All patients were keen to express their reason for consulting and reported that they had been very concerned about their cough. Patients made reference to losing sleep, having to cough all the time and worrying about a more serious infection. The duration of the cough was mentioned most often and many appeared to think a cough was unusual if it lasted more than a week, leading patients to believe they needed medical advice.

This was the first time I had such a severe cough... I usually never cough... [I] took pain killers but it did not help and after three days I decided to go to the doctor.

(Netherlands, 6)

There was some indication that patients differed in how long they had been ill before they consulted. Patients from Poland visited their GP more quickly; in addition, some patients from Poland and Belgium mentioned needing a sick note from their GP for time off work, which may have contributed to consulting earlier. Patients from other countries reported that they had usually waited around a week to consult.

Some patients were keen to justify their reasons for consulting and wanted to emphasise that they had not gone to the GP 'just like that' (Netherlands, 2). While this was common for patients from the Netherlands, England and Spain, patients from Poland and Belgium did not mention this.

Lastly, some patients delayed consulting because they knew that their usual GP did not commonly prescribe antibiotics.

I waited a week because I know [doctor] is somebody who won't prescribe antibiotics just like that.

(Belgium, 2)

2. Theme 2: Patients' perceptions of the GRACE INTRO consultation The general consultation. Most patients reported that their GP had taken time to listen to their concerns, ask questions about their illness and had given them thorough explanations about what examinations they were going to perform.

[Doctor] explained to me in detail that it could be an inflammation, but it could also be bacterial. She didn't know that yet... then said she was going to do all the tests... she did a good job.

(Netherlands, 2)

A few patients commented that their consultation had been longer than usual. This had been a result of an extended physical examination, necessary for inclusion to the trial, and/or the interventions, either doing the CRP test or explaining additional information. CRP tests had taken longer when a nurse had done them outside of the consultation, most often in England, Wales and the Netherlands. Patients were pleased to have longer consultations when their doctor had discussed their symptoms with them, but were sometimes unhappy with the time that it took to be informed of their CRP test result.
The patient booklet. Not all patients who had been offered a booklet had taken it. Some patients had felt that their GP's explanation was enough, were satisfied that their GP had acknowledged their concerns and were content that antibiotics were not required. Others said that they felt more confident when the booklet supported the treatment decision.

It is good that the doctor's words were supported by this booklet; otherwise I could have mixed thoughts about the doctor's suggestions.

(Poland, 6)

Patients with the booklet reported that they had found it easy to read and that it had provided new knowledge, most often about the duration of cough. This helped to address patients' pre-existing beliefs that they needed to consult a doctor after a week of being ill.

I know it is bronchitis and the cough can stay for three to four weeks. That is something I didn't know [before].

(Netherlands, 7)

When a GP had not discussed the booklet in the consultation some patients had then not looked at it in their own time.

I think I threw it away... I didn't keep it... if I didn't read it then, what do I need it for? I am not going to read it later.

(Spain, 10)

The CRP test. Patients appeared to be happy to have a CRP test carried out. Most described the test as something that could distinguish between a virus and bacteria, and/or indicate whether antibiotics were needed or not, better than the GP alone.

They did [the test] and said as long as it was below a certain number, it was a virus and it would be detrimental to me to take the antibiotics. If it was bad, then they would give them to me.

(Wales, 5)

Comparing interventions. Patients who received both interventions stressed that the interventions were targeted to different needs.

I think you should do both, the test as well as the booklet. Because it reassures people. You don't have to worry and that's exactly what the booklet tells you. The test tells you there is not an infection.

(Netherlands, 3)

Most patients did not have a preference between the interventions; however, those that did favoured the CRP test.

The treatment decision. Fifty-four patients (87\%) said they were satisfied with their consultation regardless of the prescribing decision. Patients felt their doctor had made a correct decision 
and had explained this decision clearly to them. Some patients reported being surprised that antibiotics had not been needed and that they had recovered without them.

Sometimes we think that if the doctor doesn't prescribe an antibiotic we are not going to get better and now I know it is not like that.

(Spain, 11)

While several had not been given antibiotics, many patients had been prescribed some medication by their GP, which appeared to have a protective effect against patients being unhappy with their consultation. This had occurred in all countries except England and Wales.

Five patients were unhappy with their consultation because they had not been given antibiotics. These patients were from England (2), Wales (2) and Poland (1), were older on average (67 years) and were from all three intervention arms.

I've been back to the doctor again this week for the same thing. I mean to my mind it's purely a means to save money on antibiotics.

(Wales, 3)

\section{Theme 3: Patients' thoughts on future illness management}

Several patients reported that they understood their illness better after consulting their doctor and that they would not be as concerned if they had a similar infection in the future. Twelve patients reported that they planned to wait for longer before consulting for a similar illness in the future. Many of these were in the trial arm with both interventions.

I will wait even longer to go to the doctor because I know [the cough] can last for longer without drawing the worst conclusions.

(Netherlands, 10)

Many patients reported that they would refer to the booklet in the future to help guide their self-management of the illness and may wait longer to consult as a result.

Five patients reported that they would re-consult for their next illness specifically to have a CRP test performed again.

I think that if I have similar symptoms again I am going to go to the consultation so that they give me the test again.

(Spain, 1)

\section{DISCUSSION}

Main findings

While two-thirds of the patients in this study had not received an antibiotic for their acute cough, the majority were happy with their consultation. Those who had received a prescription for antibiotics were satisfied they were needed and the majority of those who did not receive a prescription for antibiotics understood they were not necessary. In particular, patients who had initially expected antibiotics reported learning that they were unnecessary for their type of illness. Some patients reported learning this from the explanation given by their GP alone, but others reported that the booklet or CRP test had convinced them. This suggests that an explanation from a GP may be sufficient for some patients but that others may require additional evidence from an 'independent' source.

Patients accepted the use of GRACE INTRO interventions in their consultations. Many were pleased to be given a booklet that provided new knowledge. Equally, patients were happy for a CRP test to be used and most understood how this had contributed to the treatment decision. Patients who received both interventions emphasised that each had different uses and stated that they would want both interventions again rather than one alone.

\section{Strengths and limitations of this study}

This study provides a valuable insight into patients' perceptions of primary-care consultations using new interventions. Additionally, this study is unique as it sampled patients from different countries with varied health contexts. There did not appear to be differences in patient views of the interventions across countries and this may help explain the similar reduction of antibiotic prescribing between countries; ${ }^{19}$ however, the sampling of participants between countries was restricted by the resources in this study and sampling by intervention arm was prioritised. Future qualitative cross-country studies are needed to further examine differences in patients' views.

Obtaining socially desirable responses, by participants giving responses they think the researcher wants to hear or that they think is the 'correct' response, is a risk in all qualitative research. Here, all interviewers emphasised that they were independent from the general practice surgery, were specifically looking for views on how to improve consultations and that all responses would be kept confidential.

The number of interviewers in the study was necessary because of the multi-national context and the number of languages used to interview patients. All interviewers followed the same interview guide and provided minimal prompts. The translation of interviews from languages other than English may have led to a loss or misinterpretation of data. To ensure this was kept to a minimum, interview translations were checked by the original interviewer to ensure accuracy.

Lastly, interviewing patients in the control arm of the INTRO trial would have helped to distinguish between the views of the INTRO consultation and the views of INTRO interventions and the difference in patient satisfaction between arms. However, because the main aim was to explore the views of the interventions, the resources required to interview participants in the control arm from each country was assessed as too costly for the anticipated return.

Interpretations of findings in relation to previously published work The majority of patients were satisfied with their consultation because they were content that their GP had acknowledged their concerns and had made the correct decision. Patients had been convinced by the GP's explanation and/or the CRP test result or booklet. This supports previous research that indicates that open and clear communication between patients and clinicians and addressing patients' concerns is likely to lead to greater patient satisfaction. $^{23,24}$

A minority of patients were unhappy with their consultation because they had not received an antibiotic prescription. These patients were older and may have had less knowledge about the problems associated with antibiotic overuse. These patients were seen most often in the communication and booklet arm of the trial; however, the numbers were small. Quantitative data from a survey of INTRO patients indicated that patients in the communication and booklet arm were more likely to be satisfied with the consultation and these data provide a more representative view. ${ }^{25}$ Recent evidence has suggested that substantial proportions of patients may expect antibiotics and hope to get them in consultations. ${ }^{26,27}$ Nevertheless, it was encouraging that most patients did not expect antibiotics for acute cough and this supports previous findings that patients are now generally more knowledgeable about the appropriate use of antibiotics. ${ }^{16}$

Some patients reported that they would still consult their GP in the future within the first week of onset of a similar illness. For 
patients in Poland and Belgium this would be necessary for taking time off work because self-certification would not be possible. ${ }^{28}$ For others, this indicated that some may not have fully appreciated the information about the duration of cough and that it may take more consultations for them to understand this. This fits in with findings that show that persisting concerns drive (re)consultations for cough. ${ }^{29}$ Although some patients indicated that they would re-consult with a future illness to receive the CRP test again, recent long-term outcomes of the first trial evaluating point-of-care CRP testing show that CRP does not medicalise by driving future consultations. ${ }^{30}$ However, the same study did show that patients exposed to a GP trained in communication skills were less likely to receive antibiotics for RTI during 3.5 years of follow-up.

Implications for future research, policy and practice

The results indicate that both the interactive booklet and CRP test are interventions that are acceptable to patients for use in consultations for acute cough. GPs are likely to need practice in using these interventions to ensure that they do not disrupt consultation times and that patients get the full benefit from them. Most patients received the booklet positively although information seemed to have been retained better when GPs had discussed the booklet interactively within the consultation. Some patients mentioned the length of time taken to do the CRP test, indicating that GPs were still getting used to the equipment. Alternative, simpler versions of such tests are now available, which are likely to be quicker and easier to incorporate in practice. There were differences between countries regarding whether the GP or nurse carried out the test, which indicated that some GPs had found their own way of incorporating testing into consultations. Overall the use of both interventions appeared to help GPs explain non-prescribing decisions to patients without negatively affecting patient satisfaction.

\section{Conclusions}

Patients were satisfied with consultations that provided selfmanagement advice and reassurance but not antibiotics when consulting for acute cough. A patient booklet is an acceptable way to provide new information and advice to patients if it is discussed with a GP in the consultation. Patients prefer point-of-care CRP tests to assess whether they need antibiotics and are convinced by the results. The booklet provides information that can be referred to during a subsequent illness, and there were some indications that this, along with an explanation about self-limiting illness, may give patients the confidence to consult less.

\section{ACKNOWLEDGEMENTS}

We would like to thank all the patients and GPs who agreed to participate in the GRACE INTRO trial and in particular the patients who agreed to take part in these additional interviews.

\section{CONTRIBUTIONS}

The GRACE INTRO study was conceived by PL, SC, CCB, HG and TJMV. HG led the funding application and provided overall coordination of the GRACE consortium. All authors contributed to the development of the protocol, and to the management of the study. ST-C, SA, CB, PF-V, JK and CL carried out interviews with patients. ST-C, SA, NAF and JWC were responsible for data analysis. All authors contributed to the interpretation of the data and the writing of the paper.

\section{COMPETING INTERESTS}

The authors declare no conflict of interest.

\section{FUNDING}

This work was funded by the European Commission Framework Programme 6, the National Institute for Health Research and the Research Foundation Flanders.

\section{REFERENCES}

1 Goossens H, Ferech M, Stichele RV, Elseviers M. Outpatient antibiotic use in Europe and association with resistance: a cross-national database study. Lancet 2005; 365: 579-587.

2 van der Velden AW, Pijpers EJ, Kuyvenhoven MM, Tonkin-Crine SKG, Little P, Verheij TJM. Effectiveness of physician-targeted interventions to improve antibiotic use for respiratory tract infections. $\mathrm{Br} J$ Gen Pract 2012; 62: e801-e807.

3 Thoolen B, de Ridder D, van Lensvelt-Mulders G. Patient-oriented interventions to improve antibiotic prescribing practices in respiratory tract infections: a metaanalysis. Health Psychol Rev 2012; 6: 92-112.

4 Smith SM, Fahey T, Smucny J, Becker LA. Antibiotics for acute bronchitis. Cochrane Database Syst Rev 2004; (4): CD000245.

5 Andrews T, Thompson M, Buckley DI, Heneghan C, Deyo R, Redmond N et al. Interventions to influence consulting and antibiotic use for acute respiratory tract infections in children: a systematic review and meta-analysis. PLOS ONE 2012; 7: e30334.

6 Cals JWL, Schot MJC, de Jong SAM, Dinant GJ, Hopstaken RM. Point-of-care C-reactive protein testing and antibiotic prescribing for respiratory tract infections: a randomized controlled trial. Ann Fam Med 2010; 8: 124-133.

7 Cals JW, Butler CC, Hopstaken RM, Hood K, Dinant GJ. Effect of point of care testing for $C$ reactive protein and training in communication skills on antibiotic use in lower respiratory tract infections: cluster randomised trial. BMJ 2009; 338: b1374.

8 Butler CC, Simpson SA, Dunstan F, Rollnick S, Cohen D, Gillespie D et al. Effectiveness of multifaceted educational programme to reduce antibiotic dispensing in primary care: practice-based randomised controlled trial. BMJ 2012; 344: d8173.

9 Francis NA, Butler CC, Hood K, Simpson S, Wood F, Nuttall J. Effect of using an interactive booklet about childhood respiratory tract infections in primary care consultations on reconsulting and antibiotic prescribing: a cluster randomised controlled trial. BMJ 2009; 339: b2885.

10 Lewin S, Glenton C, Oxman AD. Use of qualitative methods alongside randomised controlled trials of complex healthcare interventions: methodological study. BMJ 2009; 339: b3496.

11 O'Cathain A, Thomas KJ, Drabble SJ, Rudolph A, Hewison J. What can qualitative research do for randomised controlled trials? A systematic mapping review. BMJ Open 2013; 3: e002889.

12 Cals JWL, Butler CC, Dinant GJ. 'Experience talks': physician prioritisation of contrasting interventions to optimise management of acute cough in general practice. Implement Sci 2009; 4: 57.

13 Cals JWL, Chappin FHF, Hopstaken RM, van Leeuwen ME, Hood K, Butler CC et al. C-reactive protein point-of-care testing for lower respiratory tract infections: a qualitative evaluation of experiences by GPs. Fam Pract 2010; 27: 212-218.

14 Tonkin-Crine S, Yardley L, Coenen S, Fernandez-Vandellos P, Krawczyk J, Touboul P et al. GPs' views in five European countries of interventions to promote prudent antibiotic use. Br J Gen Pract 2011; 61: e252-e261.

15 Brookes L, Shaw A, Sharp D, Hay AD. Towards a better understanding of patients' perspectives of antibiotic resistance and MRSA: a qualitative study. Fam Pract 2008; 25: 341-348

16 Brookes-Howell L, Elwyn G, Hood K, Wood F, Cooper L, Goossens H et al. 'The body gets used to them': patients' interpretations of antibiotic resistance and the implications for containment strategies. J Gen Intern Med 2012; 27: 766-772.

17 Wood F, Brookes-Howell L, Hood K, Cooper L, Verheij T, Goossens H et al. A multicountry qualitative study of clinicians' and patients' views of point of care tests for lower respiratory tract infection. Fam Pract 2011; 28: 661-669.

18 Francis N, Wood F, Simpson S, Hood K, Butler C. Developing an 'interactive' booklet on respiratory tract infections in children for use in primary care consultations. Patient Educ Couns 2008; 73: 286-293.

19 Little P, Stuart B, Francis N, Douglas E, Tonkin-Crine S, Anthierens S et al. Effects of internet-based training on antibiotic prescribing rates for acute respiratory-tract infections: a multinational, cluster, randomised, factorial, controlled trial. Lancet 2013; 382: 1175-1182.

20 Anthierens S, Tonkin-Crine S, Douglas E, Fernandez-Vandellos P, Krawczyk J, Llor C et al. General practitioners' views on the acceptability and applicability of a webbased intervention to reduce antibiotic prescribing for acute cough in multiple 
European countries: a qualitative study prior to a randomised trial. BMC Fam Pract 2012; 13: 101.

21 Braun V, Clarke V. Using thematic analysis in psychology. Qual Res Psychol 2006; 3: 77-101.

22 Ritchie J, Spencer L. Qualitative data analysis for applied policy research. In: Bryman A, Burgess R (eds). Analysing Qualitative Data. Routledge: London, UK, 1993, 173-194.

23 Brookes-Howell L, Wood F, Verheij T, Prout H, Cooper L, Hood K et al. Trust openness and continuity of care influence acceptance of antibiotics for children with respiratory tract infections: a four-country, primary care qualitative study. Fam Pract 2014; 31: 102-110.

24 Cabral C, Ingram J, Hay AD, Horwood J. 'They just say everything's a virus'parent's judgment of the credibility of clinician communication in primary care consultations for respiratory tract infections in children: a qualitative study. Patient Educ Couns 2014; 95: 248-253.

25 Yardley L, Douglas E, Anthierens S, Tonkin-Crine S, O'Reilly G, Stuart B et al. Evaluation of a web-based intervention to reduce antibiotic prescribing for LRT in six European countries: quantitative process analysis of the GRACE/INTRO randomised controlled trial. Implement Sci 2013; 8: 134.

26 Coenen S, Francis N, Kelly M, Hood K, Nuttall J, Little P et al. Are patient views about antibiotics related to clinician perceptions, management and outcome? A multi-country study in outpatients with acute cough. PLOS ONE 2013; 8 : e76691.
27 McNulty CAM, Nichols T, French DP, Joshi P, Butler CC. Expectations for consultations and antibiotics for respiratory tract infection in primary care: the RTI clinical iceberg. Br J Gen Pract 2013; 63: e429-e436.

28 Halvorsen PA, Wennevold K, Fleten N, Muras M, Kowalczyk A, Godycki-Cwirko M et al. Decisions on sick leave certifications for acute airways infections based on vignettes: a cross-sectional survey of GPs in Norway and Poland. Scand J Prim Health Care 2011; 29: 110-116.

29 Cals JWL, Hood K, Aaftink N, Hopstaken RM, Francis NA, Dinant GJ et al. Predictors of patient-initiated reconsultation for lower respiratory tract infections in general practice. Br J Gen Pract 2009; 59: 761-764.

30 Cals JWL, de Bock L, Beckers PHW, Francis NA, Hopstaken RM, Hood $\mathrm{K}$ et al. Enhanced communication skills and C-reactive protein point-of-care testing for respiratory tract infection: 3.5-year follow-up of a cluster randomized trial. Ann Fam Med 2013; 11: 157-164.

(1) This work is licensed under a Creative Commons AttributionNonCommercial-ShareAlike 4.0 International License. The images or other third party material in this article are included in the article's Creative Commons license, unless indicated otherwise in the credit line; if the material is not included under the Creative Commons license, users will need to obtain permission from the license holder to reproduce the material. To view a copy of this license, visit http:// creativecommons.org/licenses/by-nc-sa/4.0/

Supplemental Information accompanies the paper on the npj Primary Care Respiratory Medicine website (http://www.nature.com/npjpcrm) 